pinned down ${ }^{8}$. It was realized that the initial chemical composition of the white dwarf that undergoes a nova was a crucial determinant of the amount of ${ }^{7} \mathrm{Li}$ synthesized in the explosion; depending on the mass of its progenitor star, the white dwarf is made of either carbon and oxygen (CO novae) or oxygen and neon (ONe novae).

In CO novae, the carbon content makes the hydrogen fusion proceed faster than in $\mathrm{ONe}$ novae, owing to the operation of the $\mathrm{CNO}$ cycle of fusion reactions. Such faster evolution prevents the destruction of ${ }^{3} \mathrm{He}$ and ${ }^{7} \mathrm{Be}$ (Fig. 1), and so results in a larger production of ${ }^{7} \mathrm{Be}$ and ${ }^{7} \mathrm{Li}$. The amount of ${ }^{7} \mathrm{Li}$ produced by a CO nova corresponds to about $10^{-10}$ of the Sun's mass, but this value largely depends on the total ejected mass.

In their study, Tajitsu et al. report the detection of highly blue-shifted absorption lines of the singly ionized radioactive isotope of ${ }^{7} \mathrm{Be}$, ${ }^{7} \mathrm{Be} \mathrm{II}$, in the near-ultraviolet spectra of the CO classical nova V339 Del, between 38 and 52 days after the explosion. The spectra were obtained using the Subaru Telescope of the
National Astronomical Observatory of Japan, which delivers high spectral resolution (about 0.0052 nanometres) and so allowed the authors to tease apart the lines of ${ }^{7} \mathrm{Be}$ II from those of ${ }^{9} \mathrm{Be}$ II, both of which occur at wavelengths around $312-313 \mathrm{~nm}$.

The finding lends support to the hypothesis that the Cameron-Fowler ${ }^{7}$ Be transport mechanism is at work in novae, as predicted theoretically 40 years ago ${ }^{6}$. The observations indicate that nova V339 Del produced at least as much ${ }^{7} \mathrm{Be}$ and ${ }^{7} \mathrm{Li}$ as predicted by theory.

The implications of these results are manifold. First, they mean that novae may play a larger part in lithium production than previously thought. Second, they may increase the probability of detecting the $478-\mathrm{keV} \gamma$-ray photons emitted in the ${ }^{7} \mathrm{Be}-\mathrm{to}^{7}{ }^{7} \mathrm{Li}$ reaction ${ }^{9}$, which have remained elusive despite observational efforts made by $\gamma$-ray missions ${ }^{10,11}$. Third, and perhaps most importantly, they suggest that measurements of ${ }^{7} \mathrm{Be}$ lines in the near-ultraviolet range and within the lifetime of the element may well provide a way of estimating the contribution of novae to the lithium abundance in the Milky Way and in the Universe in its entirety.

Margarita Hernanz is at the Institute of Space Sciences, ICE (CSIC-IEEC), 08193 Cerdanyola del Vallés, Barcelona, Spain.

e-mail:hernanz@ice.csic.es

1. Tajitsu, A., Sadakane, K., Naito, H., Arai, A. \& Aoki, W. Nature 518, 381-384 (2015).

2. Burbidge, E. M., Burbidge, G. R., Fowler, W. A. \& Hoyle, F. Rev. Mod. Phys. 29, 547-650 (1957).

3. Romano, D., Matteucci, F., Molaro, P. \& Bonifacio, P. Astron. Astrophys. 352, 117-128 (1999).

4. Cameron, A. G. W. Astrophys. J. 121, 144-160 (1955).

5. Cameron, A. G. W. \& Fowler, W. A. Astrophys. J. 164, 111-114 (1971).

6. Arnould, M. \& Nørgaard, H. Astron. Astrophys. 42, 55-70 (1975)

7. Starrfield, S., Truran, J. W., Sparks, W. M. \& Arnould, M. Astrophys. J. 222, 600-603 (1978).

8. Hernanz, M., José, J., Coc, A. \& Isern, J. Astrophys. J. 465, L27-L30 (1996)

9. Clayton, D. D. Astrophys. J. 244, L97-L98 (1981).

10. Harris, M. J., Leising, M. D. \& Share, G. H. Astrophys. J. 375, 216-220 (1991).

11. Harris, M. J. et al. Astrophys. J. 563, 950-957 (2001).

\title{
EVOLUTION
}

\section{Finches sequenced}

Darwin's finches played a key part in the formulation of his theory of evolution by natural selection. They have since become an iconic model for adaptive radiation 14 species evolved from a common ancestor to occupy different niches on the Galapagos Islands, with 1 species living on Cocos Island. On page 371 of this issue, Lamichhaney et al. present the genome sequences of 120 individuals from among all 15 species and 2 close relatives (S. Lamichhaney et al. Nature 518, 371-375; 2015).

The work marks the first extensive genomic characterization of these birds. Unexpectedly, the analysis reveals that breeding between species has continued throughout their adaptation, contributing to their evolution.

The morphology of Darwin's finches has been extensively studied, with a particular focus on the diverse shapes of their beaks (pictured: Geospiza magnirostris). The authors use their rich data set to probe the genetic basis of beak shape, and identify six genomic regions that have a role in craniofacial morphology.

One region, which encodes the protein $A L X 1$, is a major player in the rapid beak-shape evolution seen both across Darwin's finches and within one species, the medium ground finch (Geospiza fortis). The function of ALX 1 is evolutionarily conserved - mutations in this gene also affect craniofacial development in humans and zebrafish. Magdalena Skipper

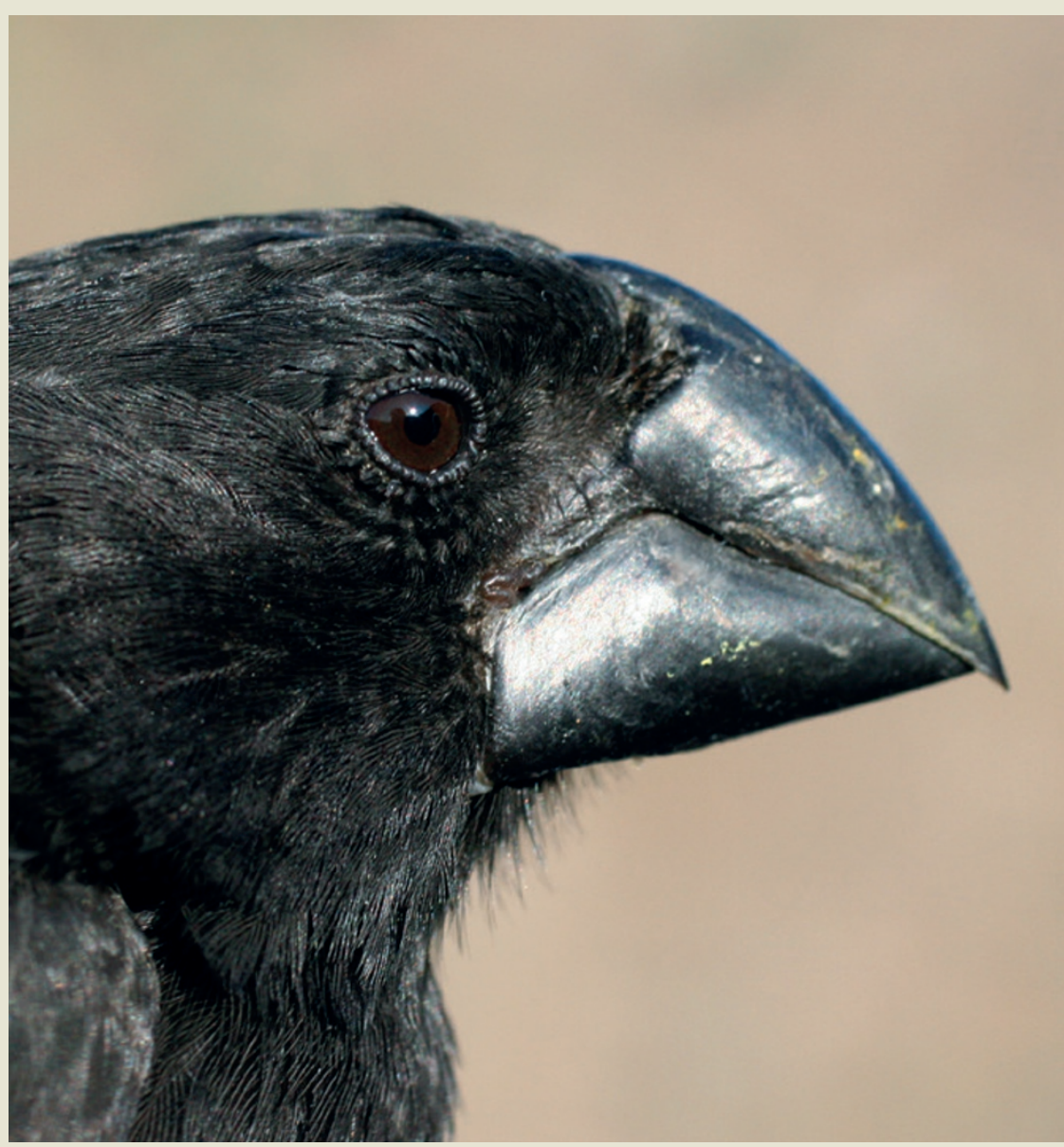

